\title{
Preface to the Issue Nonlocal Reaction-Diffusion Equations
}

\author{
M. Alfaro ${ }^{1}$, N. Apreutesei ${ }^{2}$, F. Davidson ${ }^{3}$, V. Volpert ${ }^{4}$ \\ ${ }^{1}$ I3M, University Montpellier 2, CC051, Place Eugène Bataillon, 34095 Montpellier Cedex 5, France \\ 2 Department of Mathematics, "Gheorghe Asachi" Technical University \\ Bd. Carol. I, 700506 Iasi, Romania \\ 3 Department of Mathematics, University of Dundee, Dundee, DD1 4HN, U.K. \\ ${ }^{4}$ Institut Camille Jordan, UMR 5208 CNRS, University Lyon 1, 69622 Villeurbanne, France
}

\section{Nonlocal reaction-diffusion equations in population dynamics}

Nonlocal reaction-diffusion equations are intensively studied during the last decade in relation with problems in population dynamics and other applications. In comparison with traditional reaction-diffusion equations they possess new mathematical properties and richer nonlinear dynamics. Many studies are devoted to the nonlocal reaction-diffusion equation

$$
\frac{\partial u}{\partial t}=D \frac{\partial^{2} u}{\partial x^{2}}+F(u, J(u))
$$

where

$$
F(u, J(u))=a u^{k}(1-J(u))-\sigma u, \quad J(u)=\int_{-\infty}^{\infty} \phi(x-y) u(y, t) d y,
$$

which describes the distribution of population density in the case of nonlocal consumption of resources. Here $k$ is a positive integer, $k=1$ corresponds to asexual and $k=2$ to sexual reproduction. If the kernel $\phi$ of the integral is replaced by the $\delta$-function, then we obtain conventional reaction-diffusion equation. In the case $k=1$, it is the logistic equation with the reproduction term $a u(1-u)$ proportional to the population density $u$ and to available resources $(1-u)$. In the case of nonlocal consumption of resources, the integral $J(u)$ describes consumption of resources at the space point $x$ by individuals located in some area around this point. The function $\phi(x-y)$ determines the efficiency of such consumption.

Introduction of nonlocal consumption of resources changes the properties of solutions of this equation. Consider for certainty the case where $k=1$ and $\sigma=0$. Suppose that $\int_{-\infty}^{\infty} \phi(y) d y=1$. Then $u=1$ is a stationary solution of this equation. It is stable in the case of the local equation but it can lose its stability for the nonlocal equation. If it becomes unstable, then a periodic in space stationary solution bifurcates from it [13], [19], [21]. This simple result of the linear stability analysis has important consequences from the mathematical point of view and for the applications. 
Waves. The local reaction-diffusion equation has travelling wave solutions. If $k=1$ (monostable case), travelling waves exist for all values of the speed greater than or equal to the minimal speed. In the case $k=2$ (bistable case) the wave exists for a single value of speed. The waves monotone with respect to $x$ are asymptotically stable in the corresponding norms [41].

The proof of wave existence in the case of nonlocal equation becomes much more involved, and there are only partial results [2], [5], [6], [11], [15]. The notion of generalized travelling waves, which can be characterized as propagating solutions existing for all times from $-\infty$ to $\infty$ [32], becomes appropriate here and allows the proof of wave existence without the assumption that the support of the kernel is sufficiently narrow [4], [11]. Numerical simulations show that nonmonotone travelling waves can be stable, and there exist periodic travelling waves [19], [22], [36].

Properties of solutions of the nonlocal reaction-diffusion equations are related to the spectrum of the linearized operator. The essential part of the spectrum can be found explicitly. Similarly to the usual reaction-diffusion equations, it can be completely in the left-half plane or partially in the right-half plane [7], [8], [34]. In the nonlocal case the essential spectrum can cross the imaginary axis in pure imaginary value before it crosses the origin. In this case, the periodic spatial structure emerges at infinity. Such situation is not possible for the local equation.

There are different regimes of wave propagation. First of all, there are monotone or nonmonotone waves connecting stable equilibria at infinity. If one of these equilibria loses its stability and a periodic spatial structure emerges from it due to the essential spectrum crossing the imaginary axis, then this structure propagates in space as a periodic wave. If the speed of this wave is less than the speed of the wave between two constant solutions at infinity, then they propagate one after another with the distance between them increasing in time. If the speed of the periodic wave is greater than the other one, they merge forming a single periodic wave [19], [22], [34], [36], [40]. Such behavior is observed both in the bistable and in the monostable cases.

In the monostable stable case, similar to the local case the waves are not unique. They exist for all speeds greater than or equal to the minimal speed [4]. Their structure and the patterns formed behind the waves can depend on their speed. Wave propagation is also studied in the multidimensional case [18] and for systems of equations [5], [8], [31].

Pulses. Equation (1.1) with the nonlinearity $F(u)=a u^{2}(1-u)-\sigma u$ (or with another bistable nonlinearity) can have stationary solutions decaying at infinity. Such solutions are called pulses. For the local scalar equation their existence can be easily proved. However they are unstable and, consequently, do not represent much interest for the applications. It appears that pulses can be stable in the case of global consumption, i.e., where the kernel of the integral is constant:

$$
F(u, I(u))=a u^{2}(1-I(u))-\sigma u, \quad I(u)=\int_{-\infty}^{\infty} u(y, t) d y .
$$

Their stability is observed in numerical simulations but it is not proved [34], [36].

Existence of pulses in the case of the scalar equation with global consumption can be proved analytically [34]. After that the perturbation technique allows one to prove also their existence in the case of nonlocal consumption and sufficiently large support of the kernel $\phi$ of the integral [40]. Existence of pulses is also proved for local and nonlocal monotone systems of equations [37]. The method of proof involves topological degree for elliptic problems in unbounded domains and a priori estimates of solutions. Pulses do not exist in the monostable case ( $k=1$, for constant natality and mortality coefficients).

Numerical simulations show that there is a continuous transition from periodic travelling waves to pulses for the nonlocal bistable equation. If we increase the support of the kernel of the integral, then the average speed of the periodic waves decreases. For some critical values of parameters it becomes zero, and single or multiple pulses replace the wave [36].

We can summarize the properties of local and nonlocal equations as follows. Local equations have stable monotone waves and unstable pulses. Global equations have stable pulses and do not have waves. 
Nonlocal equations have simple and periodic waves and stable or unstable pulses. Existence of stable waves and stable pulses seems to be mutually exclusive.

Let us note that there is also an extensive literature devoted to reaction-diffusion equations with time delay. It remains beyond the scope of this short review.

Applications. One of the most interesting applications of nonlocal reaction-diffusion equations concerns modelling of the emergence and evolution of biological species. If we characterize a species as a group of individuals with a close phenotype, then it can be described as a localized solution (pulse) of equation (1.1) where the space variable $x$ is some phenotypical characteristics and $u(x, t)$ is the density distribution with respect to the phenotype. As we discussed above, such stable solutions exist in the bistable case with global consumption of resources. In order to validate this approach it was used to describe human height distribution with a system of two equations with global consumption [37].

Periodic travelling waves describe the process of speciation [19], [20], [38]. They exist for both, bistable and monostable cases. The minimal model of speciation includes intraspecific competition (nonlocal consumption), reproduction and mutations (diffusion). Speciation is a general property of the living matter observed in many different contexts [35]. Other nonlocal models of natural selection, intra- and interspecific competition are discussed in [12].

One of the recent development concerns Darwinian evolution of cancer cells [14], [27], [30]. Natural selection of cancer cells can lead to the emergence of clone resistant to treatment. Moreover, this process can be stimulated by the treatment itself.

\section{About this issue}

This issue is devoted to nonlocal reaction-diffusion equations arising in various applications. It begins with the study of the zero-stabilizability for the prey population in a predator-prey system via a control which acts on predators in a subregion of the habitat [3]. The dynamics of both interacting populations is described by a reaction-diffusion system with nonlocal terms describing migrations. Conditions for the zero-stabilizability of the prey population are derived in terms of the sign of the principal eigenvalues to certain non-selfadjoint operators. A method to approximate the principal eigenvalue is presented.

A model with intraspecific competition and nonlocal dispersal instead of the usual diffusion is considered in [9]. Both interaction and dispersal are modeled using convolution integrals. It is shown that the spatially homogenous equilibrium of this model becomes unstable for sufficiently small diffusion rates by performing a linear stability analysis. Numerical observations indicate that traveling and stationary wave type patterns arise near the stability boundary. Behavior of solutions near the stability boundary is studied using the techniques of weakly nonlinear analysis. The spreading speed in the system of competition of species with nonlocal dispersion is estimated in [26]. Pattern formation in the system of competition of species with nonlocal consumption is studied in [10]. The spectrum of the linearized integro-differential operator and the decay rate of solutions is investigated in [42] (see also [7], [8]). An age structured model of bird migration is suggested in [23]. The work [17] is devoted to a nonlocal reaction-diffusion equation with random noise.

An individual based model of phenotypic trait evolution in two sex populations is considered in [43]. A macroscopic system of nonlinear differential equations describing the evolution of trait distributions in male and female subpopulations is derived passing to the limit in the number of individuals. Criteria for persistence or extinction of the population are obtained, and asymptotic stability of solution is studied.

Finally, two other papers of the issue are devoted to nonlocal equations arising in thermophysical problems of flash sintering [24] and spot welding [25]. 


\section{References}

[1] M. Alfaro, J. Coville. Rapid travelling waves in the nonlocal Fisher equation connect two unstable states. Applied Mathematics Letters, 25 (2012), 2095-2099.

[2] M. Alfaro, J. Coville, G. Raoul. Bistable travelling waves for nonlocal reaction diffusion equations. Discrete Contin. Dyn. Syst. Ser. A. 34 (2014), 1775-1791.

[3] S. Anita. Stabilization of a predator-prey system with nonlocal terms. Math. Model. Nat. Phenom., 10 (2015), no. 6, 6-16.

[4] N. Apreutesei, N. Bessonov, V. Volpert, V. Vougalter. Spatial structures and generalized travelling waves for an integro-differential equation. DCDS B, 13 (2010), No. 3, 537-557.

[5] A. Apreutesei, A. Ducrot, V. Volpert. Competition of species with intra-specific competition. Math. Model. Nat. Phenom., 3 (2008), No. 4, 1-27.

[6] N. Apreutesei, A. Ducrot, V. Volpert. Travelling waves for integro-differential equations in population dynamics. DCDS B, 11 (2009), No. 3, 541-561.

[7] N. Apreutesei, V. Volpert. Properness and topological degree for nonlocal reaction-diffusion operators. Abstract and Applied Analysis, 2011, Art. ID 629692, 21 pp.

[8] N. Apreutesei, V. Volpert. Properness and topological degree for nonlocal integro-differential systems. TMNA, 43 (2014), no. 1, 215-229.

[9] O. Aydogmus. Patterns and transitions to instability in an intraspecific competition model with nonlocal diffusion and interaction. Math. Model. Nat. Phenom., 10 (2015), no. 6, 17-29.

[10] A. Bayliss, V.A. Volpert. Patterns for competing populations with species specific nonlocal coupling. Math. Model. Nat. Phenom., 10 (2015), no. 6, 30-47.

[11] H. Berestycki, G. Nadin, B. Perthame, L. Ryzhik. The non-local Fisher-KPP equation: travelling waves and steady states. Nonlinearity, 22 (2009), no. 12, 2813-2844.

[12] N. Bessonov, N. Reinberg, V. Volpert. Mathematics of Darwin's diagram. Math. Model. Nat. Phenom., Vol. 9, No. 3, 2014, 5-25.

[13] N.F. Britton. Spatial structures and periodic travelling waves in an integro-differential reaction-diffusion population model. SIAM J. Appl. Math., 6 (1990), 1663-1688.

[14] J. Clairambault, P. Magal, V. Volpert. Cancer as evolutionary process. ESMTB Communcations, 2014, 17-20.

[15] I. Demin, V. Volpert. Existence of waves for a nonlocal reaction-diffusion equation. Math. Model. Nat. Phenom., 5 (2010), No. 5, 80-101.

[16] A. Ducrot, M. Marion, V. Volpert. Spectrum of some integro-differential operators and stability of travelling waves. Nonlinear Analysis Series A: Theory, Methods and Applications, 74 (2011), no. 13, 4455-4473.

[17] M.A. Fuentes, M.O. Caceres. Stochastic path perturbation approach applied to nonlocal nonlinear equations in population dynamics. Math. Model. Nat. Phenom., 10 (2015), no. 6, 48-60.

[18] S. Genieys, N. Bessonov, V. Volpert. Mathematical model of evolutionary branching. Mathematical and computer modelling, 49 (2009), no. 11-12, 2109-2115.

[19] S. Genieys, V. Volpert, P. Auger. Pattern and waves for a model in population dynamics with nonlocal consumption of resources. Mathem. Modelling of Natural Phenomena, 1, (2006), No. 1, 63-80.

[20] S. Genieys, V. Volpert, P. Auger. Adaptive dynamics: modelling Darwin's divergence principle. Comptes Rendus Biologies, 329 (11), 876-879 (2006).

[21] S.A. Gourley. Travelling front solutions of a nonlocal Fisher equation, J. Math. Biol., 41 (2000), $272-284$.

[22] S.A. Gourley, M.A.J. Chaplain, F.A. Davidson. Spatio-temporal pattern formation in a nonlocal reaction-diffusion equation. Dynamical systems, 16 (2001), no. 2, 173-192.

[23] S.A. Gourley, R. Liu. An age-structured model of bird migration. Math. Model. Nat. Phenom., 10 (2015), no. 6, 61-76.

[24] I.J. Hewitt, A.A. Lacey, R. I. Todd. A mathematical model for flash sintering. Math. Model. Nat. Phenom., 10 (2015), no. $6,77-89$.

[25] N.I. Kavallaris, Y. Yan. A time discretization scheme for a nonlocal degenerate problem modelling resistance spot welding Math. Model. Nat. Phenom., 10 (2015), no. 6, 90-112.

[26] L. Kong, N. Rawal, W. Shen. Spreading speeds and linear determinacy for two species competition systems with nonlocal dispersal in periodic habitats. Math. Model. Nat. Phenom., 10 (2015), no. 6, 113-141.

[27] A. Lorz et al. Populational adaptive evolution, chemotherapeutic resistance and multiple anti-cancer therapies. Mathematical Modelling and Numerical Analysis, 47 (2013), 377-399.

[28] G. Nadin, L. Rossi, L. Ryzhik, B. Perthame. Wave-like solutions for nonlocal reaction-diffusion equations: a toy model. Math. Model. Nat.Phenom., 8 (2013), no. 3, 33-41.

[29] B. Perthame, S. Genieys. Concentration in the nonlocal Fisher equation: the Hamilton-Jacobi limit. Math. Model. Nat. Phenom., 4 (2007), 135-151.

[30] F. Thomas et al. Applying ecological and evolutionary theory to cancer: a long and winding road. Evol. Appl. 6 (2013), $1-10$.

[31] B.L. Segal, V.A. Volpert, A. Bayliss. Pattern formation in a model of competing populations with nonlocal interactions. Physica D, 253 (2013), 12-22.

[32] S. Vakulenko, V. Volpert. Generalized travelling waves for perturbed monotone reaction-diffusion systems. Nonlinear Analysis. TMA, 2001 (46) 757-776. 
[33] V. Volpert. Elliptic partial differential equations. Volume 1. Fredholm theory of elliptic problems in unbounded domains. Birkhäuser, 2011.

[34] V. Volpert. Elliptic partial differential equations. Volume 2. Reaction-diffusion equations. Birkhäuser, 2014.

[35] V. Volpert. Branching and aggregation in self-reproducing systems. ESAIM: Proceedings and Surveys, 47 (2014), 116-129.

[36] V. Volpert. Pulses and waves for a bistable nonlocal reaction-diffusion equation. Applied Mathematics Letters, 44 (2015), 21-25.

[37] V. Volpert, N. Reinberg, M. Benmir, S. Boujena. On pulse solutions of a reaction-diffusion system in population dynamics Nonlinear Analysis 120 (2015), 76-85.

[38] V. Volpert, S. Petrovskii. Reaction-diffusion waves in biology. Physics of Life Reviews, 6 (2009), 267-310.

[39] V. Volpert, V. Vougalter. Emergence and propagation of patterns in nonlocal reaction-diffusion equations arising in the theory of speciation. In: "Dispersal, individual movement and spatial ecology", Eds. M. Lewis, Ph. Maini, S. Petrovskii. Springer Applied Interdisciplinary Mathematics Series, in press.

[40] V. Vougalter, V. Volpert. Existence of stationary pulses for nonlocal reaction-diffusion equations, Documenta Mathematica, 19 (2014) 1141-1153.

[41] A. Volpert, Vit. Volpert, Vl. Volpert. Traveling wave solutions of parabolic systems. Translation of Mathematical Monographs, Vol. 140, Amer. Math. Society, Providence, 1994.

[42] G. Zhao, S. Ruan. The decay rates of traveling waves and spectral analysis for a class of nonlocal evolution equations. Math. Model. Nat. Phenom., 10 (2015), no. 6, 142-162.

[43] P. Zwolenski. Trait evolution in two-sex populations. Math. Model. Nat. Phenom., 10 (2015), no. 6, $163-181$. 